Article

\title{
Stress Analysis for Cylinder Made of FGM and Subjected to Thermo-Mechanical Loadings
}

\author{
El-Sayed Habib ${ }^{\mathbb{D}}$, Medhat Awad El-Hadek ${ }^{\mathbb{D}}$ and Abla El-Megharbel * $\mathbb{C}$ \\ Production Engineering Department, Port-Said University, Port-Said 42523, Egypt; \\ elsayed.a.habib@gmail.com (E.-S.H.); melhadek@eng.psu.edu.eg (M.A.E.-H.) \\ * Correspondence: aelmegharbel@eng.psu.edu.eg; Tel.: +20-111-752-9834
}

Received: 20 November 2018; Accepted: 17 December 2018; Published: 20 December 2018

\begin{abstract}
Functionally-graded materials (FGM) have recently been widely used. Furthermore, FGM are widely recommended in cylinder design. This study represents a mathematical analysis of the stresses and strains of an FGM cylinder. The paper is interested in introducing a stress analysis to an FGM cylinder where its properties vary exponentially in the r-direction. Firstly, a benchwork has been done and compared with recent works in the same field. The radial stress, the hoop stress, and the longitudinal stress on a cylinder under mechanical and thermal loading have been theoretically derived. In addition, a finite element analysis has been investigated through ANSYS software. Finally, the values of stresses obtained from the derived equations and the corresponding values of stresses obtained from ANSYS have been compared. The obtained results established the efficiency of the proposed stress distribution and the optimization model in this research, which would be helpful for understanding well the technical design of FGM vessels or related constructions.
\end{abstract}

Keywords: thermal stress; pressure vessel; boundary effect; FGM; FE

\section{Introduction}

At the present time, functionally-graded materials (FGM) have been selected for use in many engineering applications due to their sustainability to elevated temperatures and severe temperature gradients. For these reasons, many researchers were interested in studying the behavior and properties of the functionally-graded materials. It is generally assumed that the properties of the FGM vary according to a linear equation, a power equation, or an exponential equation. Some researchers have investigated the exponential law index of FGM, which is a useful parameter from a design point of view, and can be tailored to specific applications to control the stress distributions [1].

Many researchers were interested in studying the behaviors of the FG materials in different structures. Bayat et al. [2] presented an analytical and numerical solution to achieve the spherically-symmetric thermal and mechanical stresses in a thick hollow sphere made of functionally-graded material under combined pressure and temperature loading. The material properties were assumed to be graded along the radial direction according to a power law function. A comparison between the results of the analytical findings and the numerical simulations signified that the errors were less than $0.1 \%$ of the FGM specimens. Additionally, Carrera et al. [3] addressed the static analysis of functionally-graded material plates subjected to transverse mechanical loadings. The unified formulation and principle of virtual displacements were employed to obtain both closed-form and finite element solutions. The obtained solutions (closed form and finite element) were validated through a comparison of three-dimensional exact solutions and other available solutions. Meanwhile, El-Megharbel [4] tried to introduce a theoretical analysis of functionally graded beam under thermal loading. The author assumed that the material properties changed according to a power law while the Poisson's ratio was considered constant along the beam height. Moreover, the derived formulae 
of the functionally-graded beam were compared with a traditional Timoshenko beam, resulting in good agreement. Cinefra et al. [5] considered the thermo-mechanical analysis of a simply supported, functionally-graded shell. Classical and higher-order shell theories were implemented in cases of both an equivalent single layer and layer-wise variable description by referring to Carrera's Unified Formulation. They have obtained results in a good agreement with the quasi-3D solution obtained considering mathematical layers with constant properties in the FGM layer and using high orders of expansion. Moreover, Nejad et al. [6] investigated the time-dependent thermoelastic creep response for isotropic rotating thick-walled cylindrical pressure vessels made of functionally-graded material. They assumed that material properties were a function of the radius of the cylinder and that the Poisson's ratio was constant, and the creep stresses, creep strains, and radial displacement were plotted against dimensionless radius and time for different values of the powers of the material properties. They recommended that inhomogeneity constants have a significant influence on the distributions of the creep stresses and radial displacement. You et al. [7] presented an accurate method to carry out an elastic analysis of thick-walled spherical pressure vessels subjected to internal pressure. Two kinds of pressure vessel were considered: one consisting of two homogeneous layers near the inner and outer surfaces of the vessel and one functionally graded layer in the middle; the other consisting of the functionally graded material only. In addition, the comparison with a numerical method indicated that the proposed approach converges very quickly and had excellent accuracy, whereas Kordkheili et al. [8] presented a semi-analytical solution for thermoelasticity equilibrium equations as well as for the non-linear heat transfer equation of a thin axisymmetric rotating disk made of functionally-graded materials. The effects of the radial gradation of constitutive components on stress, strain and displacement components of the FG disk had been investigated for both centrifugal force and uniform thermal loadings. It was seen that, for a given pair of materials, there was a particular volume fraction that reached extremes at a specified mechanical response under a given thermomechanical loading. In addition, it was observed that, for all values of the grading index $n$, the radial stress due to centrifugal force attained its maximum value at about $r / R_{o}=0.45$. Moreover, it was shown from the results that the property gradation correlates with thermomechanical responses of FG disks.

Furthermore, it is noble to indicate that some researchers introduced the principals of the analysis of functionally-graded materials under either thermal or mechanical loadings [9-13].

Other researchers were attracted to the FG cylinder either in the rotating case or under torsion being, or not, under thermal stress. Gharibi et al. [14] introduced an elastic analysis to the functionally-graded rotating thick cylindrical pressure vessels with exponentially-varying properties. Through a Frobenius series, profiles were plotted for various values of inhomogeneity constant and angular velocity for the radial deformation, and radial and circumferential stresses, as a function of the $r$ direction. Moreover, the research was presented with a finite element code, ANSYS, which showed excellent agreement between the analytical solutions and the solutions based on the ANSYS finite element code. The researchers investigated that the inhomogeneity constant provides a major effect on the mechanical behaviors of the exponential FG thick cylindrical vessels under pressure. On the other hand, Sharma et al. [15] investigated the creep behavior of the functionally-graded cylinder in torsion under internal and external pressure, as well as thermal loading. The influence of various parameters, such as pressure, temperature, and strain measurements on creep stresses, had been studied [9]. It has been observed that, with or without thermal effects, the cylinder made up of less functionally-graded material was on the safer side of design in torsion as compared to a cylinder made up of highly functionally-graded material and homogeneous material for linear and nonlinear strain measurements. It was observed that creep shear stresses were tensile in nature and were the maximum at the internal surface with linear and nonlinear measurements. Meanwhile, Safari et al. [16] developed an analytical method based on the Laplace transform and series solution to study the two-dimensional thermoelastic stresses in a finite-length functionally-graded thick hollow cylinder under thermal shock loading. Thermo-mechanical properties of the FG cylinder were assumed to be temperature independent and vary continuously and smoothly through the radial direction as a nonlinear power function in terms of 
volume. The effects of thermal shock loading on the dynamic characteristics of the FG thick hollow cylinder were studied in various points across the thickness of cylinder for various grading patterns of FGMs. Their results had a high capability of use in the wave propagation analysis of FG structures.

Some other researchers were concerned with the FG cylinder under thermal and mechanical stresses. Shariyat [17] presented an algorithm for nonlinear transient behavior analysis of thick functionally-graded cylindrical vessels or pipes with temperature-dependent material properties under thermo-mechanical loads. Time variations of the temperatures, displacements, and stresses were obtained through a numerical Laplace inversion. He confirmed the results from the efficiency of the algorithm and revealed the significant effects of the temperature-dependency of the material properties and the elastic wave reflections and interferences on the responses. Meanwhile, Peng et al. [18] investigated the thermoelastic analysis for axisymmetry of an FGM hollow cylinder through converting it to a Fredholm integral equation. Then, the solved equation enabled obtaining the distribution of all the stress components for the power-law gradient. Moreover, the numerical results of the radial, circumferential, and axial stresses were evaluated and presented for some typically varying material properties. Moreover, Tutuncu [19] investigated the stresses in thick-walled FGM cylinders with exponentially varying properties. The researcher obtained a five-digit accuracy by taking twenty terms in the power series. Additionally, the radial displacement distribution, the radial stress distribution, and the hoop stress distribution through the thickness was presented. The researcher concluded that the increase of the positive inhomogeneity constant caused an increase in the stress values. On the other hand, Wang et al. [20] introduced an analytical solution and FE solution for determining the mechanical behavior of the FGM pressure vessel subjected to thermo-mechanical loading. They assumed that the mechanical properties vary according to a power law. The derived analytical solution was in good agreement with the FE analysis. In addition, Alikarami and Parvizi [21] tried to introduce an elasto-plastic analysis of FG cylindrical vessels under the influence of combined pressure and temperature. They assumed that the material properties change according to a power law in the radius direction. Furthermore, the FG cylindrical vessel under pressure and temperature gradient loadings was simulated in the ABAQUS/Explicit software. The results of their theoretical analysis of the FG cylinder were compared with those of the finite element simulation and good agreements were observed between them.

However, the literature survey is lacking the exponential higher-order term analysis of cylindrical FGM under both mechanical and thermal conditions, and it is comparable to FE, which is the novelty of this work. Therefore, the aim of this work is to present an analytical solution to the stresses on a cylinder made of a functionally graded material (FGM) and subjected to thermo-mechanical loadings. In this paper, it is assumed that Young's modulus, the coefficient of thermal expansion, and thermal conductivity vary exponentially in the $r$ direction. However, the Poisson's ratio is assumed to be constant. Moreover, a finite element analysis has been conducted using ANSYS software. Finally, a comparison between the derived equations and the results from ANSYS have been conducted.

\section{Numerical Analysis}

This work presents the numerical analysis of stress and strain of a pressure vessel made of functionally-graded material subjected to both thermal and mechanical loadings. The stress analysis will consist of, first, an analytical solution to derive a mathematical representation for the stresses resulted from the applied loads using the software Mathematica; second, a finite element solution by building a 3D model expressing the grading characteristic of the material and applied loads through ANSYS software (Version 15.0.7, Ansys Inc., Canonsburg, PA, USA); and, finally, compare the corresponding stresses to confirm the results.

The analytical solution procedure will take two steps; the foremost step is selecting a benchmark with the aid of Wang et al. [20], while the material properties of the cylinder change through the thickness (in the $r$-direction) according to a power law. Moreover, the second step applies the same 
analytical procedure on a cylinder that its material properties change through the thickness according to an exponential equation.

\subsection{Benchmarking for Analysis}

In this section, a stress analysis is presented for a pressure vessel made of functionally-graded material and subjected to both thermal and mechanical loadings with material properties varying according to the power law, using Mathematica, and the resulting stress formulations and their relative values with those obtained by Wang et al. [20], which has been used as a benchmark for the solution methodology, are compared.

\subsubsection{Derivation of Analytical Solution}

It is assumed that the material of the FG pressure vessel is graded according to a power law in the following forms:

$$
\alpha(r)=\alpha_{o}\left(\frac{r}{R_{o}}\right)^{\eta} \quad E(r)=E_{o}\left(\frac{r}{R_{o}}\right)^{\zeta} \quad \lambda(r)=\lambda_{o}\left(\frac{r}{R_{o}}\right)^{\psi}
$$

where $r, E, \alpha$, and $\lambda$ are the radial radius, Young's modulus, coefficient of thermal expansion, and thermal conductivity, respectively. The symbols $R_{0}, E_{0}, \alpha_{0}$, and $\lambda_{0}$ are the radial radius, Young's modulus, coefficient of thermal expansion and thermal conductivity of the outside wall of the cylinder, respectively, while, $\zeta, \eta$, and $\psi$ are the graded factors of the FGM pressure vessel. Whereas, the Poisson's ratio is assumed to be constant.

Under the linear-elastic uncoupled thermo-mechanical conduction condition, the combined stresses will be the summation of both mechanical and thermal stresses for radial, hoop, and axial stresses (i.e., $\sigma_{r}(r)=\sigma_{M r}(r)+\sigma_{T r}(r)$ ). The coupling effect between the mechanical stress and thermal stress was ignored to reduce the difficulty of solving complex equations.

The pressure vessel is assumed to be under mechanical load in the form of internal pressure $P$ and thermal loads because of the temperature variation on both sides of the pressure vessel wall, where $T_{i}$ is the internal temperature and $T_{o}$ is the external temperature.

The temperature distribution model is expressed by the steady-state equation as follows:

$$
\frac{\partial}{\partial r}\left(r \lambda(r) \frac{\partial}{\partial r} T(r)\right)=0
$$

where $T(r)$ is the radial function of the temperature along the wall thickness direction which can be deduced using Equation (2) by applying the thermal boundary conditions on both sides of the wall, which are $T(r)=T_{i}$ at $r=R_{i} \& T(r)=T_{o}$ at $r=R_{o}$ and can be written as follows:

$$
T(r)=\frac{R_{o}^{-\psi} T_{i}-R_{i}^{-\psi} T_{o}+r^{-\psi}\left(-T_{i}+T_{o}\right)}{-R_{i}^{-\psi}+R_{o}^{-\psi}}
$$

For the uncoupled thermo-elastic steady-state condition problem, the constitutive equations of the pressure vessel subjected to the mechanical and thermal loads are given as:

$$
\begin{aligned}
\sigma_{r}(r) & =\frac{E(r)}{1+v}\left(\epsilon_{r}(r)+\frac{v}{1-2 v}\left(\epsilon_{r}(r)+\epsilon_{\theta}(r)+\epsilon_{z}\right)\right)-\left(\frac{\alpha(r) E(r) T(r)}{1-2 v}\right) \\
\sigma_{\theta}(r) & =\frac{E(r)}{1+v}\left(\epsilon_{\theta}(r)+\frac{v}{1-2 v}\left(\epsilon_{r}(r)+\epsilon_{\theta}(r)+\epsilon_{z}\right)\right)-\left(\frac{\alpha(r) E(r) T(r)}{1-2 v}\right) \\
\sigma_{z}(r) & =\frac{E(r)}{1+v}\left(\epsilon_{z}+\frac{v}{1-2 v}\left(\epsilon_{r}(r)+\epsilon_{\theta}(r)+\epsilon_{z}\right)\right)-\left(\frac{\alpha(r) E(r) T(r)}{1-2 v}\right)
\end{aligned}
$$


According to the infinitesimal strain theory, the equilibrium equation and the strain displacement relations for an axisymmetric structure can be written, respectively, as:

$$
\begin{gathered}
\frac{\partial \sigma_{r}(r)}{\partial r}+\frac{\sigma_{r}(r)-\sigma_{\theta}(r)}{r}=0 \\
\epsilon_{r}(r)=\frac{\partial u(r)}{\partial r} \quad \epsilon_{\theta}(r)=\frac{u(r)}{r} \quad \epsilon_{z}=\frac{\partial w(z)}{\partial z}
\end{gathered}
$$

where $\sigma_{r}(r), \sigma_{\theta}(r)$ and $\sigma_{z}(r)$ are the radial, hoop, and axial stresses, respectively, as a function of $r . \epsilon_{r}(r)$ and $\epsilon_{\theta}(r)$ are the radial and hoop strain, respectively, as a function of $r$, while $\epsilon_{z}$ is the axial strain and is assumed to be constant, taking the large length-radius ratio into consideration, and whereas $u(r)$ is the radial displacement as a function of $r$ and $w(z)$ is the axial displacement as function of length. $v$ is the Poisson's ratio. In Equation (5), the body force is ignored.

The mechanical and thermal stresses will be deduced separately by applying the equilibrium equation and the strain displacement relations Equations (5) and (6) and combine with Equations (1) and (4) to obtain the governing equation in the cylindrical part in the form of the Euler-Cauchy equation for which the characteristic equation is $m^{2}+\beta m+\left(\frac{v}{1-v} \beta-1\right)=0$ and the roots of that equation are $m_{1}=\frac{1}{2}\left(-\zeta-\sqrt{4+\zeta^{2}-\frac{4 \zeta v}{1-v}}\right)$ and $m_{2}=\frac{1}{2}\left(-\zeta+\sqrt{4+\zeta^{2}-\frac{4 \zeta v}{1-v}}\right)$

Thus, the solution of the equation can be obtained which expresses the general equation for the displacement $u(r)$, and then the constitutive Equations (4) can be derived as a function of $r$ for both the mechanical and thermal analysis.

To obtain the mechanical and thermal solutions, the following boundary conditions are applied, respectively:

- $\quad$ First, for the mechanical loads:

$$
\begin{gathered}
\sigma_{r}^{M}=0 \quad \text { when } \quad r=R_{o} \\
\sigma_{r}^{M}=-P \quad \text { when } \quad r=R_{i} \\
\int_{R_{i}}^{R_{o}} \sigma_{z}^{M} 2 \pi r d r=P \pi R_{i}^{2}
\end{gathered}
$$

- $\quad$ Second, for the thermal loads:

$$
\begin{gathered}
\sigma_{r}^{T}=0 \quad \text { when } \quad r=R_{i} \\
\sigma_{r}^{T}=0 \quad \text { when } \quad r=R_{o} \\
\int_{R_{i}}^{R_{o}} \sigma_{z}^{T} 2 \pi r d r=0
\end{gathered}
$$

By applying the boundary conditions Equations (7) and (8), the integration's constants and the axial strain can be deduced. Thus, the analysis of the mechanical and thermal equations can be obtained. Both the benchmarked equations in Wang et al. [20] and the derived equations by Mathematica are written, respectively, in the next section.

\subsubsection{The Benchmarked Equations}

Wang et al. [20] derived the mechanical stresses and the axial strain under the condition of FGM in Equation (1). Where $\sigma_{r}^{M}, \sigma_{\theta}^{M}$, and $\sigma_{z}^{M}$ are the mechanical stresses in the radial, hoop, and axial directions, respectively. $\sigma_{r}^{T}, \sigma_{\theta}^{T}$ and $\sigma_{z}^{T}$ are the thermal stresses in the radial, hoop, and axial directions, respectively. 
- The mechanical stresses and axial strain equations

The authors in [20] had assumed that the pressure vessel was subjected only to the internal pressure, thus $T_{i}=T_{0}$ and, consequently, $T(r)=0$, to obtain the mechanical solution. By combining Equations (1), (4)-(6), besides applying the boundary conditions from Equation (7), the mechanical radial, hoop, and axial stresses and the axial strain could be obtained [20].

- The thermal stresses and axial strain equations

By combining Equations (1), (3)-(6), and applying the boundary conditions Equation (8) the thermal radial, hoop, and axial stresses and the axial strain can be obtained [20].

\subsubsection{The Analytical Model}

Here the authors derived the corresponding previous equations through Mathematica software (Version 11.2.0.0, Wolfram Research of Champaign, Champaign, IL, USA). Both mechanical and thermal equations are derived below.

- The mechanical stresses and axial strain equations

The same solution procedure is used as stated above, the pressure vessel is subjected only to the internal pressure, thus $T_{i}=T_{0}$ and, consequently, $T(r)=0$. By combining Equations (1), (4)-(6), and applying the boundary conditions from Equation (7), the mechanical radial, hoop, and axial stresses and the axial strain can be derived through Mathematica software and written as follows:

$$
\begin{gathered}
\sigma_{r}^{M}(r)_{D}=\frac{\left(\left(r^{m_{1}} C_{M 1}\left(-v+(-1+v) m_{1}\right)+r^{m_{2}} C_{M 2}\left(-v+(-1+v) m_{2}\right)\right)\left(\frac{r}{R_{o}}\right)^{\zeta} E_{o}\right)}{(r(1+v)(-1+2 v))} \\
\sigma_{\theta}^{M}(r)_{D}=-\frac{\left(r^{m_{1}} C_{M 1}\left(1-v+v m_{1}\right)+r^{m_{2}} C_{M 2}\left(1-v+v m_{2}\right)\right)\left(\frac{r}{R_{o}}\right)^{\zeta} E_{o}}{r(1+v)(-1+2 v)} \\
\sigma_{z}^{M}(r)_{D}=\frac{-\left(\left(\left(\frac{r}{R_{o}}\right)^{\zeta}\left(r^{m_{1}} v C_{M 1}\left(1+m_{1}\right)+r^{m_{2}} v C_{M 2}\left(1+m_{2}\right)-r\left(-1+v+2 v^{2}\right) \epsilon_{M z D}\right) E_{o}\right)\right)}{(r(1+v)(-1+2 v))}
\end{gathered}
$$

where $\epsilon_{M z D}, C_{M 1}$, and $C_{M 2}$ are constants.

- The thermal stresses and axial strain equations

By combining Equations (1), (3)-(6), and applying the boundary conditions from Equation (8), the thermal radial, hoop, and axial stresses and the axial strain can be derived and written as follows:

$$
\begin{aligned}
\sigma_{r}^{T}(r)_{D}= & \left(\frac{r}{R_{o}}\right)^{\zeta}\left(\frac { 1 } { - 1 + v + 2 v ^ { 2 } } r ^ { - 1 - \psi } \left(r ^ { 1 + \eta } \left((-1+\eta(-1+v)+\psi-v \psi) C_{T 3}\right.\right.\right. \\
& \left.+r^{\psi}(-1+\eta(-1+v)) C_{T 4}\right)+r^{\psi+m_{1}} C_{T 1}\left(-v+(-1+v) m_{1}\right) \\
& \left.+r^{\psi+m_{2}} C_{T 2}\left(-v+(-1+v) m_{2}\right)\right) \\
& \left.+\frac{\left(\frac{r}{R_{o}}\right)^{\eta}\left(-T_{o}+\left(\frac{r}{R_{o}}\right)^{-\psi}\left(\frac{R_{i}}{R_{o}}\right)^{\psi}\left(\left(-1+\left(\frac{r}{R_{o}}\right)^{\psi}\right) T_{i}+T_{o}\right)\right) \alpha_{o}}{(-1+2 v)\left(-1+\left(\frac{R_{i}}{R_{o}}\right)^{\psi}\right)}\right) E_{o}
\end{aligned}
$$




$$
\begin{aligned}
\sigma_{\theta}^{T}(r)_{D}= & \left(\frac{r}{R_{o}}\right)^{\zeta}\left(\frac { 1 } { - 1 + v + 2 v ^ { 2 } } r ^ { - 1 - \psi } \left(-r^{1+\eta}\left((1+\eta v-v \psi) C_{T 3}+r^{\psi}(1+\eta v) C_{T 4}\right)\right.\right. \\
& \left.-r^{\psi+m_{1}} C_{T 1}\left(1-v+v m_{1}\right)-r^{\psi+m_{2}} C_{T 2}\left(1-v+v m_{2}\right)\right) \\
& +\frac{\left(\frac{r}{R_{o}}\right)^{\eta}\left(-T_{o}+\left(\frac{r}{R_{o}}\right)^{-\psi}\left(\frac{R_{i}}{R_{o}}\right)^{\psi}\left(\left(-1+\left(\frac{r}{R_{o}}\right)^{\psi}\right) T_{i}+T_{o}\right)\right) \alpha_{o}}{(-1+2 v)\left(-1+\left(\frac{R_{i}}{R_{o}}\right)^{\psi}\right)} E_{o} \\
\sigma_{z}^{T}(r)_{D}= & \left(\frac{r}{R_{o}}\right)^{\zeta}\left(\frac{\left(\frac{r}{R_{o}}\right)^{\eta}\left(-T_{o}+\left(\frac{r}{R_{o}}\right)^{-\psi}\left(\frac{R_{i}}{R_{o}}\right)^{\psi}\left(\left(-1+\left(\frac{r}{R_{o}}\right)^{\psi}\right) T_{i}+T_{o}\right)\right) \alpha_{o}}{(-1+2 v)\left(-1+\left(\frac{R_{i}}{R_{o}}\right)^{\psi}\right)}\right. \\
& +\frac{1}{-1+v+2 v^{2}} r^{-1-\psi}\left(r^{1+\eta} v\left((-2-\eta+\psi) C_{T 3}-r^{\psi}(2+\eta) C_{T 4}\right)\right. \\
& +r^{\psi}\left(-r^{m_{1}} v C_{T 1}\left(1+m_{1}\right)-r^{m_{2}} v C_{T 2}\left(1+m_{2}\right)+r(-1+v\right. \\
& \left.\left.\left.\left.+2 v^{2}\right) \epsilon_{T z D}\right)\right)\right) E_{o}
\end{aligned}
$$

where $\epsilon_{T z D}, C_{T 1}, C_{T 2}, C_{T 3}$, and $C_{T 4}$ are constants.

\subsubsection{Outcomes}

As a case study, the previous analytical solution is investigated for a cylinder with cross-section shown in Figure 1. The internal radius of the cylinder is $R_{i}=800 \mathrm{~mm}$, the external radius is $R_{o}=840 \mathrm{~mm}$, and the length of the cylinder is $1600 \mathrm{~mm}$. The cylindrical vessel is subjected to an internal pressure of $10 \mathrm{MPa}$, a temperature of $120{ }^{\circ} \mathrm{C}$ on the inner surface, and a temperature of $20{ }^{\circ} \mathrm{C}$ on the outer surface. Two primary materials are chosen for analysis $\mathrm{Al}_{2} \mathrm{O}_{3}$ for ceramic and stainless steel for metal with $E_{o}=210 \mathrm{GPa}, v=0.30$, and the grade factors are $\zeta=-6.4024, \eta=7.2554$, and $\psi=35.5406$.

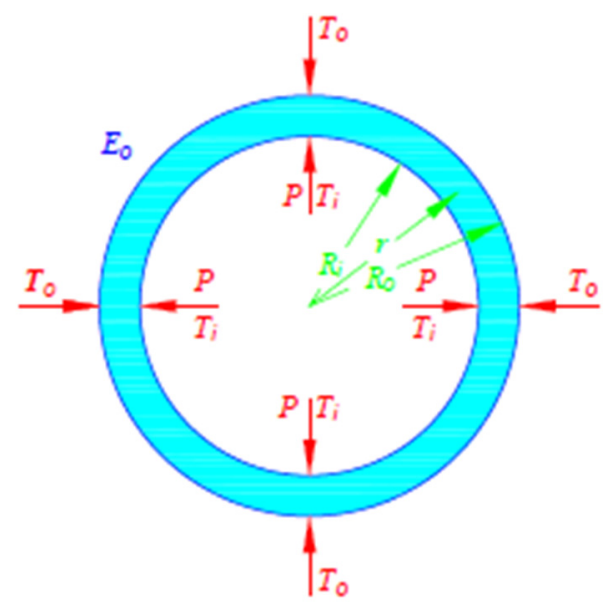

Figure 1. The cross-section of the functionally graded pressure vessel subjected to internal pressure and thermal loadings.

As shown in Figures 2-4, the determined values for the analytical solution of the derived radial, hoop and axial stresses equations (obtained through Mathematica) with that of the benchmarked radial, hoop, and axial stress equations obtained from Wang et al. [20] are compared versus the radius. 


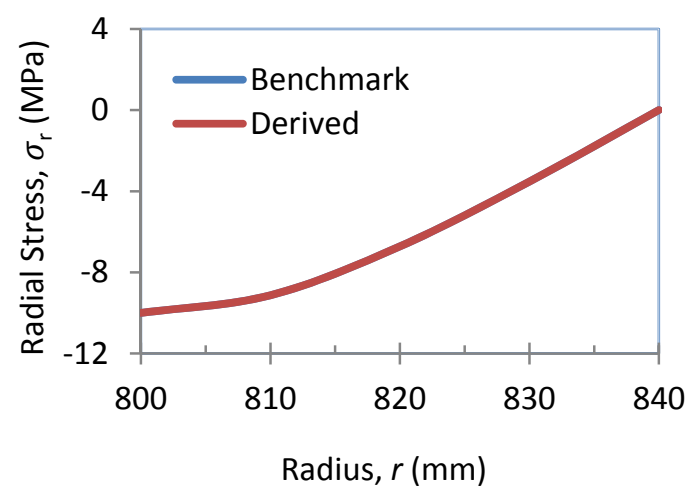

Figure 2. The variation of radial stress with cylinder radius.

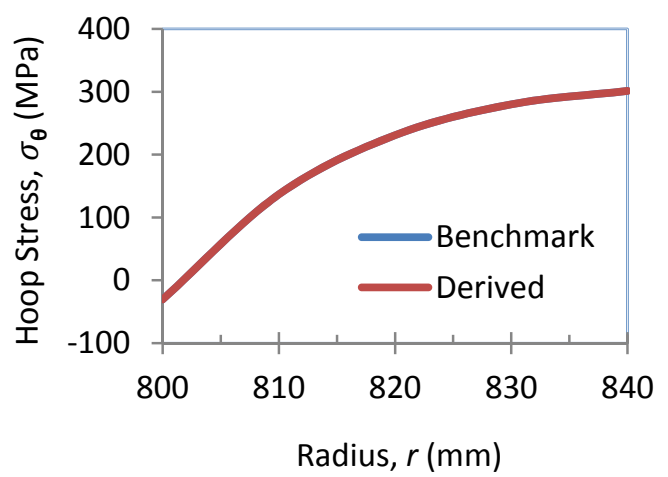

Figure 3. The variation of hoop stress with cylinder radius.

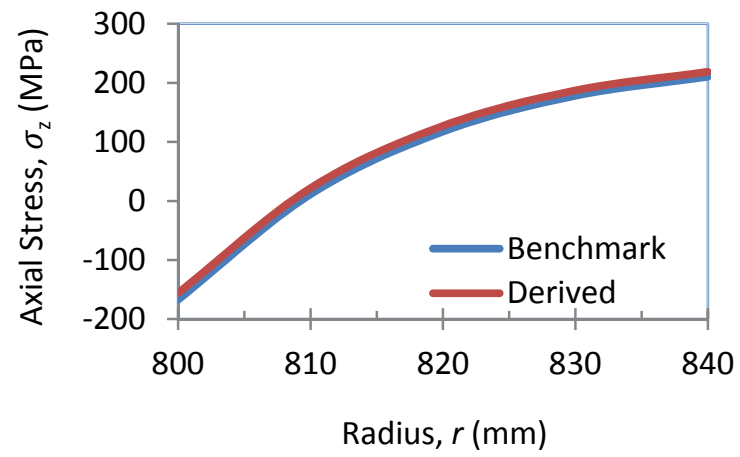

Figure 4. The variation of axial stress with cylinder radius.

As depicted in Figures 2 and 3, the derived values for the radial and hoop stresses and the corresponding benchmark equations [20] match perfectly for both the radial and hoop stresses. On the other hand, the axial stress, shown in Figure 4, has a slight difference with a percentage less than 5\%, which is satisfactory.

\subsection{Analytical Solution for an Exponentially-Graded FG Vessel}

\subsubsection{Derivation of the Analytical Solution}

In this study, the mechanical and thermal stresses will be derived for a cylindrical vessel subjected to internal pressure and thermal loading on the internal surface of the cylinder. An exponential form is constructed to express the mechanical properties variation through the cylinder thickness. A coupled thermo-elastic steady-state conduction problem is assumed, and by substituting with the constitutive equations of the pressure vessel subjected to the mechanical and thermal loads and applying the boundary conditions to solve the equilibrium equation and the strain displacement theory for an 
axisymmetric structure according to the infinitesimal strain theory to get the general equations for the stresses and axial strain.

It is assumed that the material of the FG pressure vessel is graded according to an exponential relationship as per the following forms:

$$
E(r)=E_{o} e^{\beta\left(1-\frac{r}{R_{0}}\right)} \quad \alpha(r)=\alpha_{o} e^{\varphi\left(1-\frac{r}{R_{o}}\right)} \quad \lambda(r)=\lambda_{o} e^{\gamma\left(1-\frac{r}{R_{o}}\right)}
$$

where $\beta, \varphi$ and $\gamma$ are the graded factors of the FGM pressure vessel, and the Poisson's ratio is assumed to be a constant.

Under the linear-elastic uncoupled thermo-mechanical conduction condition, the combined stresses will be the summation of both mechanical and thermal stresses for radial, hoop, and axial stresses (i.e., $\sigma_{r}(r)=\sigma_{M r}(r)+\sigma_{T r}(r)$ ). The coupling effect between the mechanical stress and thermal stress was ignored to reduce the difficulty of solving complex equations.

The temperature distribution function can be deduced using Equation (2) by applying the thermal boundary conditions on both sides of the wall which are $T(r)=T_{i}$ at $r=R_{i}$ and $T(r)=T_{o}$ at $r=R_{o}$ and can be written as follows:

$$
T(r)=\frac{e^{-\gamma} K_{1} E i\left(\frac{r \gamma}{R_{o}}\right)}{\lambda_{o}}+K_{2}
$$

For the uncoupled thermo-elastic steady-state condition problem, the constitutive equations of the pressure vessel subjected to the mechanical and thermal loads are given as per Equation (4).

According to the infinitesimal strain theory, the equilibrium equation and the strain displacement relations for an axisymmetric structure Equations (5) and (6) shall be applied.

The mechanical and thermal stresses will be deduced separately by applying the equilibrium equations to obtain the governing equations in the cylindrical part in the form of a second order linear nonhomogeneous differential equation with variable coefficients which by solving will give the general equation for the displacement $u(r)$, then the constitutive equations can be derived as a function of $r$, the same boundary conditions as per the benchmark to be applied; and Equations (7) and (8) to obtain the integration's constants and the axial strain equations.

Thus, the analysis of the mechanical and thermal equations can be listed as follows:

- Mechanical equations and axial strain equations

The mechanical stresses and axial strain equations can be written as follows:

$$
\begin{aligned}
\sigma_{M r}(r)= & \frac{1}{r(1+v)(-1+2 v)} e^{\beta-\frac{r \beta}{R_{o}}} E_{o}\left(a_{M 1}\left(-v f_{M 1}[r]+r(-1+v) f_{M 1}^{\prime}[r]\right)\right. \\
& +\epsilon_{M z}\left(-v\left(r+f_{M 2}[r]\right)+r(-1+v) f_{M 2^{\prime}}[r]\right)+a_{M 2}\left(-v f_{M 3}[r]\right. \\
& \left.\left.+r(-1+v) f_{M 3^{\prime}}[r]\right)\right)
\end{aligned}
$$

where, $\epsilon_{M z}, a_{M 1}$, and $a_{M 2}$ are constants.

$f_{M i}[r]$ are functions of $r$ used for simplification of stress equations.

- Thermal equations and axial strain equations 
The Thermal stresses and axial strain equations can be written as follows:

$$
\begin{aligned}
& \sigma_{T r}(r)=-\frac{e^{\beta\left(1-\frac{r}{R_{o}}\right)+\varphi\left(1-\frac{r}{R_{o}}\right)} \alpha_{o} E_{o}\left(K_{2}+\frac{e^{-\gamma} E i\left(\frac{r \gamma}{R_{o}}\right) K_{1}}{\lambda_{o}}\right)}{1-2 v} \\
& +\frac{1}{1+v} e^{\beta\left(1-\frac{r}{R_{0}}\right)} E_{o}\left(K_{1} f_{T 1}{ }^{\prime}[r]+K_{2} f_{T 2}{ }^{\prime}[r]+\epsilon_{T z} f_{T 3}{ }^{\prime}[r]+a_{T 1} f_{T 4}{ }^{\prime}[r]\right. \\
& +a_{T 2} f_{T 5}{ }^{\prime}[r]+\frac{1}{1-2 v} v\left(\epsilon_{T z}\right. \\
& +\frac{K_{1} f_{T 1}[r]+K_{2} f_{T 2}[r]+\epsilon_{T z} f_{T 3}[r]+a_{T 1} f_{T 4}[r]+a_{T 2} f_{T 5}[r]}{r} \\
& \left.\left.+K_{1} f_{T 1}{ }^{\prime}[r]+K_{2} f_{T 2}{ }^{\prime}[r]+\epsilon_{T z} f_{T 3}{ }^{\prime}[r]+a_{T 1} f_{T 4}{ }^{\prime}[r]+a_{T 2} f_{T 5}{ }^{\prime}[r]\right)\right) \\
& \sigma_{T \theta}(r)=-\frac{e^{\beta\left(1-\frac{r}{R_{o}}\right)+\varphi\left(1-\frac{r}{R_{o}}\right)} \alpha_{o} E_{o}\left(K_{2}+\frac{e^{-\gamma} E i\left(\frac{r \gamma}{R_{o}}\right) K_{1}}{\lambda_{o}}\right)}{1-2 v} \\
& +\frac{1}{1+v} e^{\beta\left(1-\frac{r}{R_{0}}\right)} E_{o}\left(\frac{K_{1} f_{T 1}[r]+K_{2} f_{T 2}[r]+\epsilon_{T z} f_{T 3}[r]+a_{T 1} f_{T 4}[r]+a_{T 2} f_{T 5}[r]}{r}\right. \\
& +\frac{1}{1-2 v} v\left(\epsilon_{T z}+\frac{K_{1} f_{T 1}[r]+K_{2} f_{T 2}[r]+\epsilon_{T z} f_{T 3}[r]+a_{T 1} f_{T 4}[r]+a_{T 2} f_{T 5}[r]}{r}\right. \\
& \left.\left.+K_{1} f_{T 1}{ }^{\prime}[r]+K_{2} f_{T 2}{ }^{\prime}[r]+\epsilon_{T z} f_{T 3^{\prime}}[r]+a_{T 1} f_{T 4}{ }^{\prime}[r]+a_{T 2} f_{T 5^{\prime}}[r]\right)\right) \\
& \sigma_{T z}(r)=-\frac{e^{\beta\left(1-\frac{r}{R_{o}}\right)+\varphi\left(1-\frac{r}{R_{o}}\right)} \alpha_{o} E_{o}\left(K_{2}+\frac{e^{-\gamma} \operatorname{Ei}\left(\frac{r \gamma}{R_{o}}\right) K_{1}}{\lambda_{o}}\right)}{1-2 v}+\frac{1}{1+v} e^{\beta\left(1-\frac{r}{R_{o}}\right)} E_{o}\left(\epsilon_{T z}\right. \\
& +\frac{1}{1-2 v} v\left(\epsilon_{T z}\right. \\
& +\frac{K_{1} f_{T 1}[r]+K_{2} f_{T 2}[r]+\epsilon_{T z} f_{T 3}[r]+a_{T 1} f_{T 4}[r]+a_{T 2} f_{T 5}[r]}{r} \\
& \left.\left.+K_{1} f_{T 1}{ }^{\prime}[r]+K_{2} f_{T 2}{ }^{\prime}[r]+\epsilon_{T z} f_{T 3}{ }^{\prime}[r]+a_{T 1} f_{T 4}{ }^{\prime}[r]+a_{T 2} f_{T 5}{ }^{\prime}[r]\right)\right)
\end{aligned}
$$

where $\epsilon_{T z}, a_{T 1}$, and $a_{T 2}$ are constants.

$f_{T i}[r]$ are functions of $r$ used for the simplification of the stress equations.

\subsubsection{FE Modelling}

As the cylinder is subjected to both thermal and mechanical loadings, a thermo-mechanical coupled FE model is constructed to verify the analytical solution. The model is a cylinder (Figure 5) with internal radius of $R_{i}=800 \mathrm{~mm}$, external radius of $R_{o}=840 \mathrm{~mm}$, and length of $1600 \mathrm{~mm}$. Since there is no authorized element in all existing FE codes to simulate the mechanical behavior of FGM, and although some scholars used a stepwise composition-based FE model, in this work the FGM mechanical properties are implemented within the program as a function changing with the change of temperature to discretize the exponential gradient properties of the material with a more accurate manner. ANSYS was used as a solver and post-processing tool due to its conjunction with a powerful mathematical package with good symbolic and numerical calculation capabilities in the form of function visualization [22,23]. A full finite element model for the cylinder is shown in Figure 6a, Considering the symmetry of the cylinder geometry and loads as well as boundary conditions, a quarter of the FG vessel is constructed, as shown in Figure 6b, with an eight-node 3D thermal element SOLID 70 which has a three-dimensional, steady-state, or transient thermal analysis capability and an eight-node 3D structural element SOLID185, which has plasticity, stress stiffening, 
large deflection, and large strain capabilities using ANSYS. The boundary conditions are applied, and the model meshed into 16,960 elements and 19,602 nodes. As the stresses vary through the cylinder thickness (the radial direction), a finer meshing is applied for the thickness to be able to obtain more accurate results, as shown in Figure 6c. The vessel is subjected to an internal pressure of $10 \mathrm{MPa}$. The temperature of the inner surface is $120^{\circ} \mathrm{C}$, and the temperature of the outer surface is $20^{\circ} \mathrm{C}$. Two primary materials are considered: $94 \%$ alumina for ceramic, and stainless steel 304 for metal, where $E_{o}=200 G P a, v=0.29$, and the grade factors are $\beta=8.514767, \varphi=-15.93569$, and $\gamma=2.21257$.

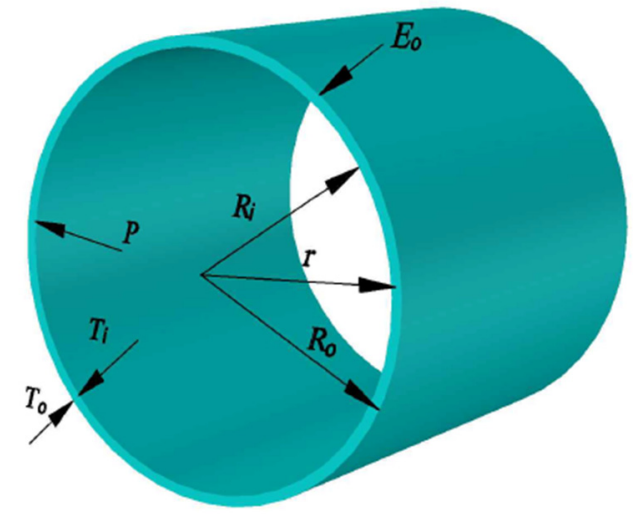

Figure 5. Cylinder model with geometrical parameters and applied loads.

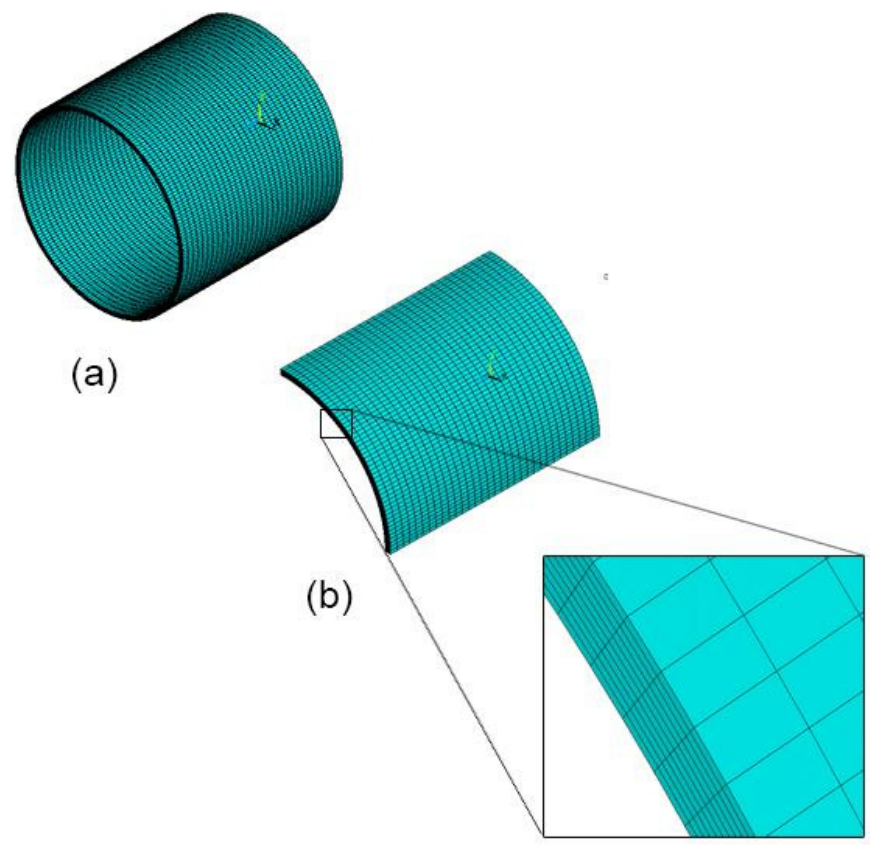

(c)

Figure 6. (a) Full FE model of the cylinder, (b) quarter axisymmetric model of meshed elements, and (c) magnified segment of the meshed elements showing the fine meshing in the radial direction.

\subsubsection{Results}

Figures 7 and 8 illustrate the comparison between the analytical and FE results for both radial, and hoop axial stresses, as well as for the analytical SS or ceramic. There is a significant difference between SS and FGM analytical results, as shown in Figure 7 or Figure 8.

The error between the FE and the analytical results is about $10 \%$ as explained in Figures 7 and 8 , as the analysis conducted by the FEM is limited due to the numerical accuracy of the software. 
A good agreement is found for the axial stress for both the analytical and the FE solution, as shown in Figure 9. Additionally, Figure 10 shows the comparison of von Mises values for both analytical and FE results which gives an error of $12 \%$.

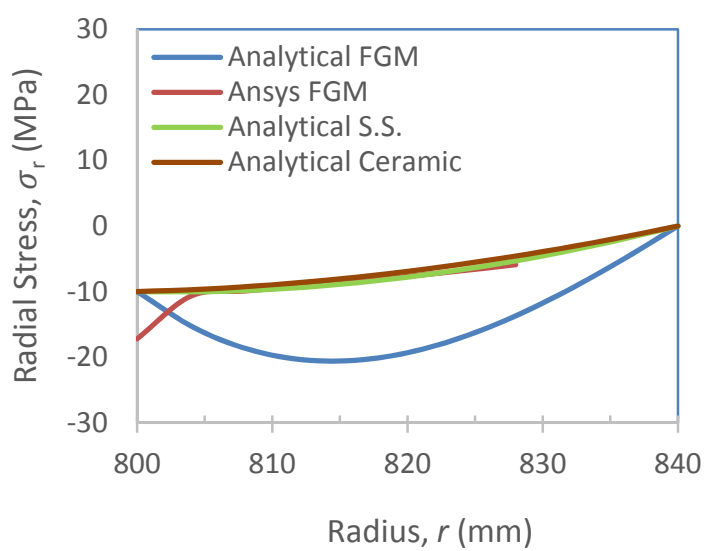

Figure 7. The variation of radial stress with cylinder radius.

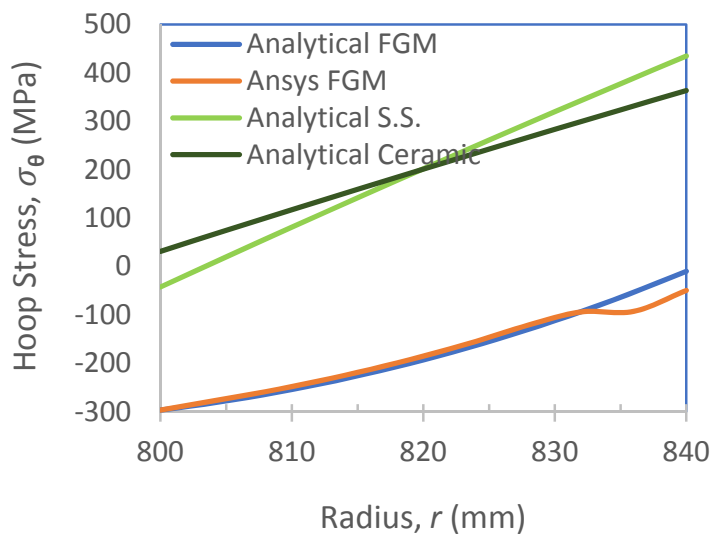

Figure 8. The variation of hoop stress with cylinder radius.

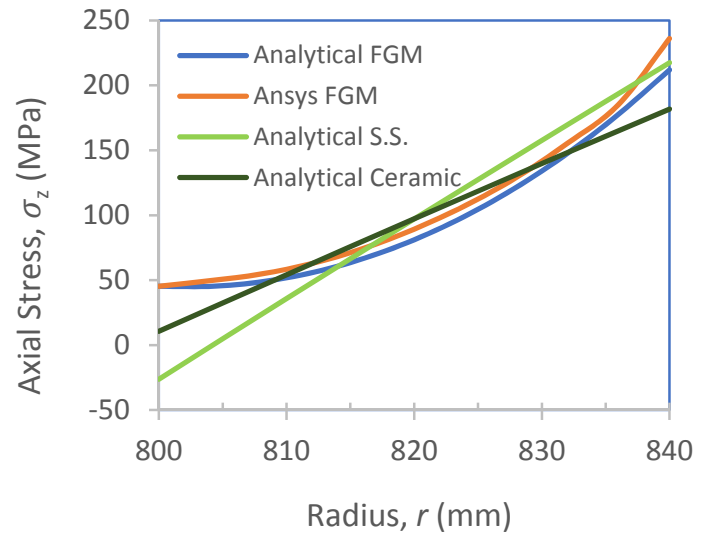

Figure 9. The variation of axial stress with cylinder radius. 


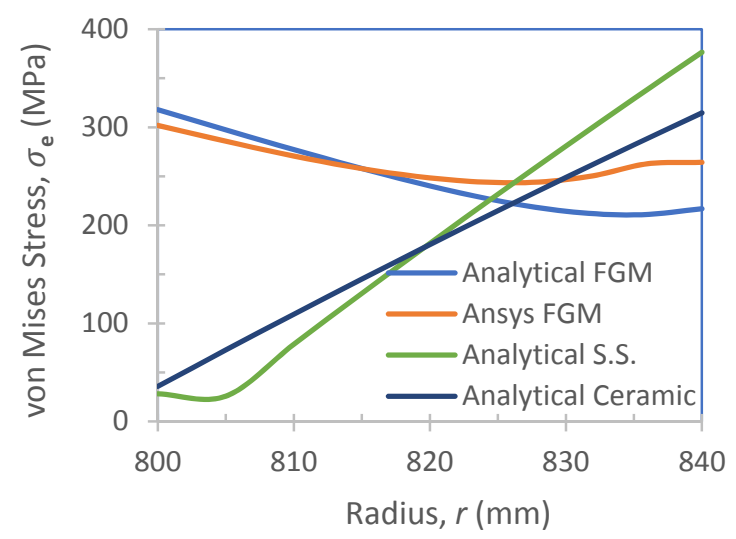

Figure 10. The variation of von Mises stress with cylinder radius.

\section{Conclusions}

To investigate the stresses resulting due to the applyication of thermal and mechanical loadings on a pressure vessel made of functionally-graded material, whose mechanical properties vary according to an exponential function, the following steps were followed.

A benchmark for the analytical solution for a pressure vessel with the mechanical properties variation following a power law as in [13] was conducted with the help of mathematical software, and the results gave a very good match.

The mathematical software is used and, by following the benchmark procedure, the analytical solution for our case of FGM with exponentially-varying properties is achieved.

The results have been discussed numerically and described graphically. An FE model is constructed and solved using the ANSYS software package to verify the analytical solution for our case, and although ANSYS has no authorized element in all existing FE codes to simulate the mechanical behaviour of FGM, a procedure was followed to build an FE model with the material exponentially graded.

Both the analytical and the FE results are compared and found agreement between the results. Additionally, the presented outcomes illustrate that the inhomogeneity constant provides a significant effect on the mechanical behaviors of the exponential FG cylindrical under pressure and thermal stresses.

Author Contributions: Formal analysis: M.A.E.-H. and A.E.-M.; Investigation: E.-S.H. and A.E.-M.; Methodology: A.E.-M.; Software: E.-S.H., M.A.E.-H., and A.E.-M.; Supervision: M.A.E.-H.; Writing-Original Draft: E.-S.H.; Writing-Review and Editing: A.E.-M.

Funding: This is a self-supported research without funding from any agency whatsoever.

Conflicts of Interest: The authors declare that there is no conflict of interest regarding the publication of this paper.

\section{References}

1. Rasheedat, M.M.; Esther, T.A. Functionally Graded Materials; Carlos, P.A., Bergmann, P., Eds.; Springer International Publishing: Cham, Switzerland, 2017.

2. Bayat, Y.; Ghannad, M.; Torabi, H. Analytical and numerical analysis for the FGM thick sphere under combined pressure and temperature loading. Arch. Appl. Mech. 2012, 82, 229-242. [CrossRef]

3. Carrera, E.; Brischetto, S.; Robaldo, A. Variable Kinematic Model for the Analysis of Functionally Graded Material plates. AIAA J. 2008, 46, 194-203. [CrossRef]

4. El-Megharbel, A. A Theoretical Analysis of Functionally Graded Beam under Thermal Loading. World J. Eng. Technol. 2016, 4, 437-449. [CrossRef]

5. Cinefra, M.; Carrera, E.; Brischetto, S.; Belouettar, S. Thermo-mechanical analysis of functionally graded shells. J. Therm. Stress. 2010, 33, 942-963. [CrossRef] 
6. Nejad, M.Z.; Kashkoli, M.D. Time-dependent thermo-creep analysis of rotating FGM thick-walled cylindrical pressure vessels under heat flux. Int. J. Eng. Sci. 2014, 82, 222-237. [CrossRef]

7. You, L.H.; Zhang, J.J.; You, X.Y. Elastic analysis of internally pressurized thick-walled spherical pressure vessels of functionally graded materials. Int. J. Press. Vessels Pip. 2005, 82, 347-354. [CrossRef]

8. Kordkheili, S.A.H.; Naghdabadi, R. Thermoelastic analysis of a functionally graded rotating disk. Compos. Struct. 2007, 79, 508-516. [CrossRef]

9. Srividhya, S.; Raghu, P.; Rajagopal, A.; Reddy, J. Nonlocal nonlinear analysis of functionally graded plates using third-order shear deformation theory. Int. J. Eng. Sci. 2018, 125, 1-22. [CrossRef]

10. Čanađija, M.; Barretta, R.; de Sciarra, F.M. On Functionally Graded Timoshenko Nonisothermal Nanobeams. Compos. Struct. 2016, 135, 286-296. [CrossRef]

11. Čanađija, M.; Barretta, R.; de Sciarra, F.M. A gradient elasticity model of BernoullieEuler nanobeams in non-isothermal environments. Eur. J. Mech. A Solids 2016, 55, 243-255. [CrossRef]

12. De Sciarra, F.M.; Salerno, M. On thermodynamic functions in thermoelasticity without energy dissipation. Eur. J. Mech. A Solids 2014, 46, 84-95. [CrossRef]

13. Barretta, R.; Canađija, M.; Luciano, R.; de Sciarra, F.M. Stress-driven modeling of nonlocal thermoelastic behavior of nanobeams. Int. J. Eng. Sci. 2018, 126, 53-67. [CrossRef]

14. Gharibi, M.; Nejad, M.Z.; Hadi, A. Elastic analysis of functionally graded rotating thick cylindrical pressure vessels with exponentially-varying properties using power series method of Frobenius. J. Comput. Appl. Mech. 2017, 48, 89-98.

15. Sharma, S.; Yadav, S.; Sharma, R. Thermal creep analysis of functionally graded thick- walled cylinder subjected to torsion and internal and external pressure. J. Solid Mech. 2017, 9, 302-318.

16. Safari, A.; Tahani, M.; Hosseini, S.M. Two-dimensional dynamic analysis of thermal stresses in a finite-length FG thick hollow cylinder subjected to thermal shock loading using an analytical method. Acta Mech. 2011, 220, 299-314. [CrossRef]

17. Shariyat, M. A nonlinear Hermitian transfinite element method for transient behavior analysis of hollow functionally graded cylinders with temperature-dependent materials under thermo-mechanical loads. Int. J. Press. Vessels Pip. 2009, 86, 280-289. [CrossRef]

18. Peng, X.L.; Li, X.F. Thermoelastic analysis of a cylindrical vessel of functionally graded materials. Int. J. Press. Vessels Pip. 2010, 87, 203-210. [CrossRef]

19. Tutuncu, N. Stresses in thick-walled FGM cylinders with exponentially-varying properties. Eng. Struct. 2007, 29, 2032-2035. [CrossRef]

20. Wang, Z.W.; Zhang, Q.; Xia, L.Z.; Wu, J.T.; Liu, P.Q. Stress Analysis and Parameter Optimization of an FGM Pressure Vessel Subjected to Thermo-Mechanical Loadings. Procedia Eng. 2015, 130, 374-389. [CrossRef]

21. Alikarami, S; Parvizi, A. Elasto-plastic analysis and finite element simulation of thick-walled functionally graded cylinder subjected to combined pressure and thermal loading. Sci. Eng. Compos. Mater. 2017, 24, 609-620. [CrossRef]

22. El-Hadek, M.A. Numerical Simulation of the Inertia Friction Welding Process of Dissimilar Materials. Metall. Mater. Trans. B 2014, 45, 2346-2356. [CrossRef]

23. El-Hadek, M.A. Dynamic equivalence of ultrasonic stress wave propagation in solids. Ultrasonics 2018, 83, 214-221. [CrossRef] [PubMed]

(C) 2018 by the authors. Licensee MDPI, Basel, Switzerland. This article is an open access article distributed under the terms and conditions of the Creative Commons Attribution (CC BY) license (http://creativecommons.org/licenses/by/4.0/). 